\title{
Human Cytomegalovirus Envelope Glycoprotein B
}

National Cancer Institute

\section{Source}

National Cancer Institute. Human Cytomegalovirus Envelope Glycoprotein B. NCI

Thesaurus. Code C132331.

Envelope glycoprotein B (906 aa; 102 kDa) is encoded by the human cytomegalovirus gB gene, which is located within the open reading frame (ORF) UL55. This protein is involved in host cell entry, cell to-cell virus transmission, and fusion of infected cells. 